\title{
Surface Plasmons on Metamaterials
}

\author{
Alastair P. Hibbins*, Matthew J. Lockyear, J. Roy Sambles \\ Electromagnetic Materials Research Group, School of Physics, University of Exeter, Stocker Road, \\ Exeter EX4 4QL, United Kingdom.
}

\begin{abstract}
The field of plasmonics has historically been a playground exclusively for the optics community. Primarily this is because the response of metals becomes dominated by their large conductivities at much lower frequencies, making it difficult to exploit the unique properties of surface plasmon (SP) modes. Indeed SPs on flat, perfectly conducting substrates are better described as simple surface currents or grazing photons. However the realization that one can form metaldielectric composites to support surface waves with plasmon-like properties has opened the field of plasmonics to the terahertz and microwave domains. Pendry et al. [Science, 305, 847 (2004)] were among the first to speculate about an extension of plasmonics into long wavelength regimes. They demonstrated that the perforated surface of a perfect conductor can support a SP-like mode whose behavior is determined purely by the geometry of the substrate. Beginning with our initial experimental verification of these SP-like modes excited via grating-coupling, we present an overview of some of our recent microwave studies. We progress to study the classical method of prism coupling and also consider the enhanced transmission phenomenon (mediated by plasmon-like surface modes) through hole arrays. Finally the first experimental evidence of coupled SP-like modes between two such perforated metal substrates placed in close proximity will be presented.
\end{abstract}

Keywords: surface plasmons, surface waves, diffraction, gratings, metamaterials, microwave, hole arrays, waveguides

\section{INTRODUCTION}

The electromagnetic surface waves discussed in this work propagate along an interface between two dissimilar media without radiation losses. Surface waves [1,2] have attracted much scientific attention over the last one hundred years. For example, Zenneck [3] in 1907 realized the possibility of propagation of radio waves around the Earth when one considers one half-space as a pure dielectric and the other as a conductor. A similar wave of radiation coupled to oscillating charge density may propagate along a metal-dielectric interface. We refer to this mode as a surface plasmon (SP) and its influence on the reflectivity from metallic gratings may be realized in the visible part of the spectrum as a Wood's anomaly [4-7]. Because the real parts of the dielectric functions of the media either side of the interface are of opposite signs, the normal components of the electric field at the metal-dielectric interface oppose each other. Hence the surface mode is strongly localized at the interface with exponentially decaying fields into the adjacent media. However, the dispersion relation of a SP on a planar surface [5] is such that the wave vector of the mode is always greater than the maximum photon wave vector available in the adjacent dielectric. Hence, on a flat surface of infinite extent, the SP is unable to radiate or be directly coupled to by incident planar waves. Resonant interaction of electromagnetic radiation with the surface may only be achieved by increasing the wave vector of the incident radiation, possibly by using a high-index prism $[8,9]$ or through surface roughness. The latter is most commonly found in the form of a diffraction grating, where the periodic corrugation provides the necessary in-plane wave-vector enhancement in multiples of the grating wave-vector.

Many workers have extensively studied grating-coupled SPs at visible [5,10] and infrared [11] wavelengths on corrugated surfaces produced by standard interferographic techniques [12]. Before this method of grating manufacture was established, gratings had to be ruled by hand [4], hence experimentalists often made use of the larger scale of systems available in the microwave regime. For example, in 1957, Meecham and Peters [13] studied the reflection of $32 \mathrm{~mm}$ wavelength radiation from an echelette grating with the electric field of the radiation parallel to the grooves. As they scanned the angle of incidence, they observed a redistribution of energy among the diffracted beams at the point at which an order begins, or ceases to propagate - the pseudocritical edge or Rayleigh anomaly [14]. They compared their experimental reflectivities from a finite grating to a theory which, like many other diffraction theories developed around

*a.p.hibbins@exeter.ac.uk; phone 441392 264170; fax 441392 264111; newton.ex.ac.uk/research/emag/ 
this time, assumed the surface to be of infinite extent and perfectly conducting i.e. $\operatorname{Im}(\varepsilon)=\infty$. This is a valid assumption to make since the frequency of the impinging microwave radiation is many orders of magnitude less than the plasma frequency, and dissipative effects therefore dominate. Hence at these wavelengths, metals may often (but not always, see Ref. 15) be treated as near perfectly conducting. In 1965, Palmer et al [16] reported measurements for the spectral distribution of radiation of $4 \mathrm{~mm}$ wavelength from metal gratings of rectangular profile. When the wave propagation vector was lying in a plane normal to the grating grooves, with the electric field vector perpendicular to the grooves, dark anomalies were observed that became wider as the groove depth was increased. An attempt was made to explain this phenomenon using a theory based on "surface wave modes which are supported by the rectangular periodic structure of the grating", however no illustration of these results is presented. We believe that work provided the first experimental evidence of the propagation of SPs on near-perfectly conducting surfaces, a point that we returned to study in 1999 [17]. However three years prior to our work, Pendry et al. [18] devised a metamaterial comprised of a periodic lattice of very thin metallic wires that exhibited plasmonic behavior in the $\mathrm{GHz}$ regime analogous to that exhibited by a solid metal in the ultra-violet. And in 2004 Pendry et al. $[19,20]$ demonstrated that bound SP-like waves can be sustained even on a perfectly conducting substrate, as long as it is periodically corrugated. This seemed quite counterintuitive at the time since the fields associated SPs at microwave frequencies are known to penetrate only $\sim 1 \mu \mathrm{m}$ into the metal, while extending many hundreds of wavelengths into the dielectric above (Fig. 1a). However, perforate the metal with subwavelength holes and strongly localized SP-like waves are once again supported. This is because the holes may allow some of the field to penetrate into the substrate, hence changing the field-matching situation at the surface (Fig. 1b). The holes in the metal act as waveguides and therefore have a cutoff frequency below which no propagating modes are allowed. Hence, below cutoff, only evanescent fields exist on the metal side of the interface, and it is exactly this field characteristic that is required for a surface mode. Pendry and co-workers showed that if the size and spacing of the surface patterning is much smaller than the wavelength then the electromagnetic response of the surface can be described using an effective medium dielectric function of the Drude form, with the effective plasma frequency dictated purely by the design of the surface geometry. The cutoff frequency of the modes in the waveguides is equivalent to an effective surface plasma frequency of the structured surface, which for holes of square cross section $(a \times a)$ and infinite depth is given by

$$
v_{\mathrm{sP}}^{(h=\infty)}=\frac{c}{2 a \sqrt{\varepsilon_{\mathrm{n}} \mu_{\mathrm{n}}}}
$$

where $c$ is the velocity of light in vacuum and $\varepsilon_{\mathrm{h}}$ and $\mu_{\mathrm{h}}$ are the relative permittivity and permeability of the material filling the holes, respectively. Hence this concept of 'designer' surface modes promises the ability to engineer a plasmonlike surface wave at almost any frequency below the natural surface plasma frequency of a metal.

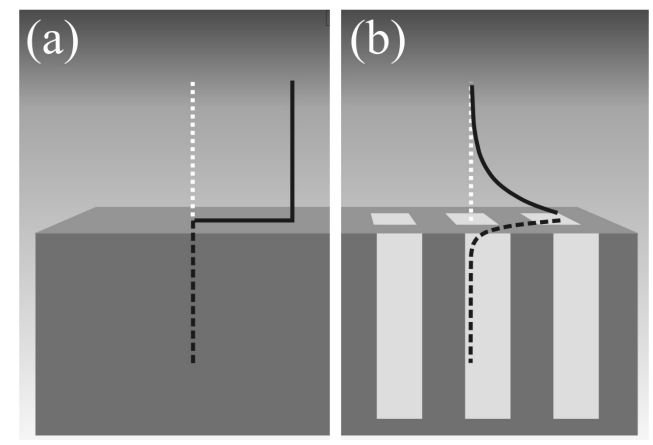

Fig. 1. Schematic representation of electric fields associated with a mode propagating along the surface of a metal. (a) At microwave frequencies, the metal is almost perfectly conducting. (b) An effective penetration depth is achieved by perforating the substrate with an array of subwavelength holes.

Here we present an overview of some of our recent experimental studies involving plasmon-like surface waves on these metamaterial surfaces in the microwave regime. Beginning with our initial experimental verification of a grating-coupled plasmon-like surface mode on a near perfectly conducting substrate, we progress to study the classical method of prism coupling. We also consider the enhanced transmission phenomenon through hole arrays, and confirm the crucial role of SP-like modes in the mechanism responsible. Furthermore, the first experimental evidence of coupled SP-like modes between two such perforated metal substrates placed in close proximity will be presented. 


\section{EXPERIMENTAL VERIFICATION OF PLASMON-LIKE SURFACE WAVES ON METALS IN THE MICROWAVE REGIME}

\subsection{Diffractive coupling}

Our sample is a $300 \mathrm{~mm} \times 300 \mathrm{~mm}$ array of hollow, square-ended brass tubes of height $h=45 \mathrm{~mm}$, side length $d=9.525 \mathrm{~mm}$ and inner dimension $a=6.960 \mathrm{~mm}$. From Eq. $1, v_{\mathrm{SP}}=21.54 \mathrm{GHz}$. The tubes are carefully arranged, square face down, on a flat brass plate and tightly clamped together. A modification of the originally proposed structure [19] is the addition of a periodic array of cylindrical rods with pitch $2 d$ positioned on the surface of the array of tubes (Fig. 2a). These allow for control of the strength of diffractive coupling to the mode supported by the surface. In addition, relying on the $d$-periodicity alone would only allow for observation of the surface mode either close to the waveguide cutoff, or at very high angles of incidence. The former could result in confusion with the onset of propagating waveguide modes, while the latter is not experimentally favorable. However, the first-order " $2 d$ " diffracted light lines associated with the rods will cross the frequency axis at $\sim 15.7 \mathrm{GHz}$. Since a band gap in the dispersion of the surface mode will be established at this crossing (normal incidence), the mode's resonant frequency will be reduced below this point. For this reason, the reflectivity features associated with the surface mode cannot be confused with the onset of diffraction.
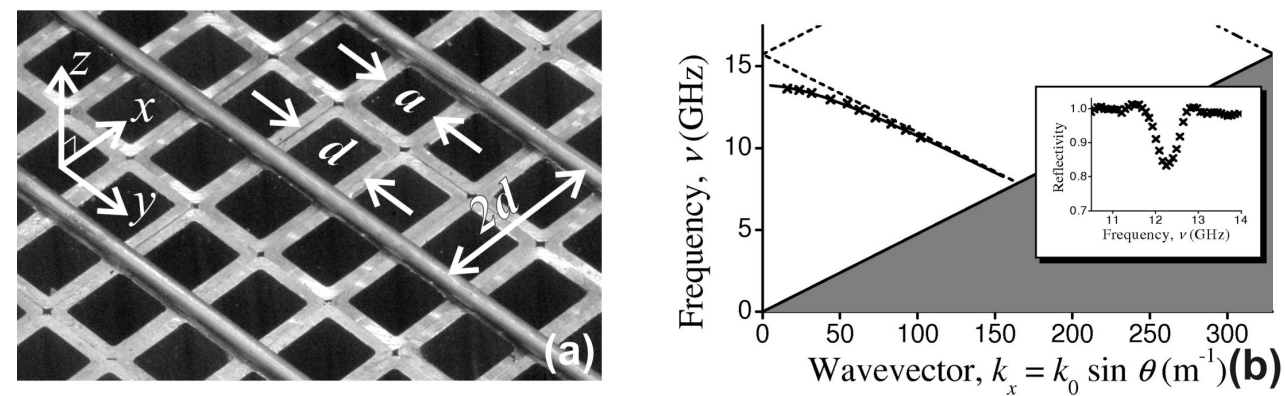

Fig. 2. (a) Photograph of experimental sample. (b) The dispersion of the surface mode on the air-filled sample. Radiation is TM-polarized and incident in the $x z$-plane. The frequency of the resonance is derived from experimental $(\times)$ and modeled (solid line) data sets. The shaded region corresponds to momentum space within which surface modes may not be directly coupled to, as they are beyond the light line. The dashed line represents the first-order diffracted light associated with the array of cylindrical rods. The dot-dashed line similarly corresponds to first-order diffraction from the array of brass tubes. (Inset) TM-polarized reflectivity spectrum obtained at $\theta \approx 14^{\circ}$, illustrating the resonant surface mode at $\sim 12.3 \mathrm{GHz}$.

Our initial set of reflectivity measurements are recorded at a series of fixed angles of incidence $(\theta)$ with a collimated microwave beam incident in the $x z$-plane [17]. The inset of Fig. $2 \mathrm{~b}$ illustrates a typical experimental reflectivity spectrum. The main part of Fig. $2 \mathrm{~b}$ shows the distribution of the resonant mode positions derived from the experimental and modeled reflectivity spectra as a function of in-plane momentum $\left(k_{x}=2 \pi v \sin \theta / c\right)$ and frequency. Verification of the plasmon-like character of the mode is provided by examining the finite element method (FEM) [Ansoft HFSS] modeled field distributions at the resonant frequency. Consider the resonance of the mode shown in the inset of Fig. 2b. Fig. 3a shows the time-averaged electric field strength with the instantaneous electric field vector distribution (plotted at a time in phase corresponding to maximum enhancement) superimposed. Regions of strongest field are clearly located directly above the metal regions of the surface, and the field line loops are highly reminiscent of the SP fields on metals. Fig. 3c further strengthens the SP argument, illustrating exponentially decaying electric field strength away from the surface. The Poynting vector plot (Fig. 3b) shows that the power flow is strongly enhanced just above the surface.

The asymptotic approach to $\sim v_{\mathrm{SP}}$ can easily be demonstrated by rotating the plane of incidence by $90^{\circ}$ (i.e., the $y z$-plane) and filling the tubes with a dielectric, hence separating the photonic band gap effect from the plasmonic asymptote [21]. However note that there is a small difference between the value of $v_{\mathrm{SP}}$ predicted by Eq. 1 and that experimentally observed (Fig. 4) since the former does not take into account reflections from each end of the finite length cavity. This results in an additional quantization requirement in the $z$-direction, destroying the continuum of waveguide modes. By naively assuming that the waveguide modes are entirely confined to the cavity, we can imagine that the effective surface plasma frequency has increased to be equal to the frequency of the lowest energy standing wave resonance, 


$$
v_{\mathrm{SP}}=c / 2 a \varepsilon_{\mathrm{h}}^{1 / 2} \cdot \sqrt{1+\left(a^{2} / 4 h^{2}\right)} .
$$

We shall return to make this approximation more exact in a subsequent paper [22].

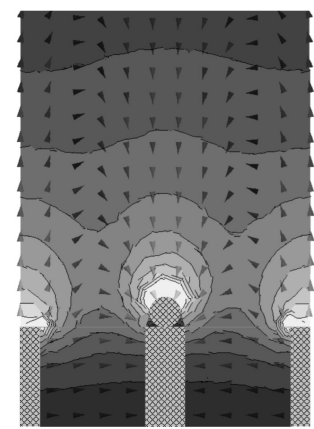

(a)

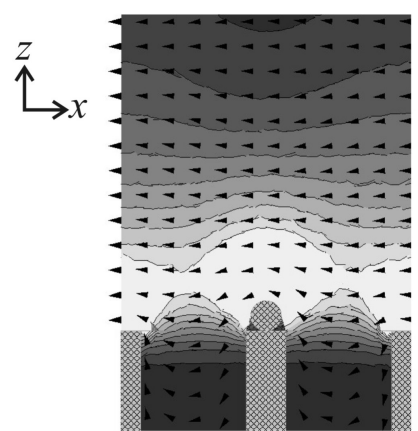

(b)

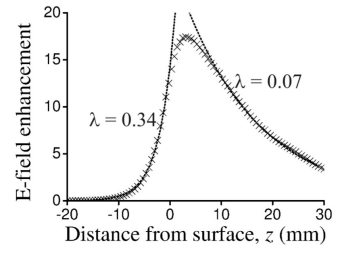

(c)

Fig. 3. Modeled fields on resonance of the mode seen in Fig. 2b inset. See main text for details. (a) Electric field strengths, (b) Poynting vector. White areas correspond to enhancement in electric field strength by a factor of $>40$ and of power flow by a factor of 300. (c) Time-averaged electric field strength calculated for either side of the sample surface at the centre of a tube waveguide (i.e. at a perpendicular distance of $a / 2$ from the inside walls). The decays on either side of the peak have been fitted to single exponentials ( $\lambda$, decay constant).

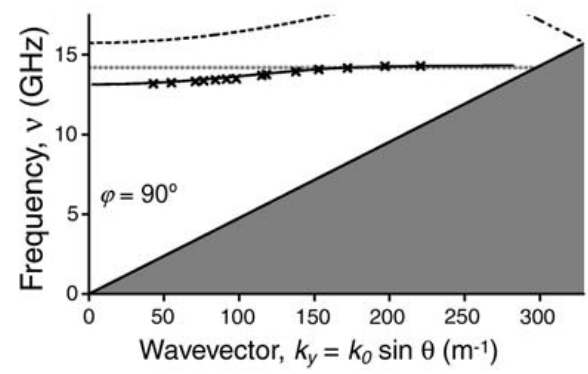

Fig. 4. The dispersion of the surface mode on the wax-filled brass tube array. Radiation is TM-polarized and incident in the $y z$-plane $\left(\varphi=90^{\circ}\right)$. The frequency of the resonance is derived from experimental $(\times)$ and modeled (solid line) data sets. The shaded region corresponds to momentum space within which surface modes may not be directly coupled to, as they are beyond the light line. The dashed lines represent the first-order diffracted light lines associated with the array of cylindrical rods. The dot-dashed lines similarly correspond to first-order diffraction from the square array of brass tubes. The horizontal dotted lines correspond to $v_{\mathrm{SP}}$ give by Eq. 1 .

\subsection{Prism coupling}

As described above, the original experimental corroboration of the existence of the plasmon-like surface waves was via grating coupling [21], rather like the observations of Wood's [4] in the visible domain. However the ultimate test of this new idea is to use, as did Otto [9], simple prism coupling. By recording the largely unperturbed dispersion curve, Otto was able to compare for the first time his experimental dispersion with that derived analytically. Here we use a large wax prism, of refractive index $n=1.5$, to couple collimated microwave radiation on to the surface of a sample similar to that shown in Fig. 2a, but with $h=15 \mathrm{~mm}$ and without the cylindrical rods (Fig. 5a). For TM-polarized incident radiation and a suitable tunnel barrier (established by spacing the prism some $t$ millimeters from the sample), with the microwave beam incident beyond the critical angle $\theta_{\text {int }}>\sin ^{-1} 1 / n$, the evanescent fields beyond the prism will resonantly couple to the plasmon-like mode on the surface of the array, and a minimum will be recorded in the reflected spectrum. By varying the spacing between the prism and the sample and recording the frequency of the reflectivity minimum, the inplane wavevector of the mode $\left(k_{/ /}\right)$may be extracted. Repetition of this measurement for different incident angles allows for a good approximation of the largely unperturbed surface wave dispersion to be derived. The onset of diffraction at the Brillouin Zone boundary is $V_{\text {diff } 10\}}=c / 2 d=15.7 \mathrm{GHz}$, and in order to satisfactorily separate the diffractive effects 
(photonic band gap) from the plasmonic-like behaviour, it is necessary for the effective surface plasma frequency $v_{\mathrm{SP}}$ to be significantly suppressed below $v_{\text {diff }}$. This is achieved by firstly filling the tubes with wax $\left(\varepsilon_{\mathrm{h}}=2.29\right)$ to lower $v_{\mathrm{SP}}$ to approximately $14 \mathrm{GHz}$. We then rotate the plane of incidence by $45^{\circ}$ around the $z$-axis so that it lies along one diagonal axis of the square tubes. Thereby we increase the onset of diffraction to $v_{\text {diff } 111}=c / \sqrt{2} d=22.3 \mathrm{GHz}$ at the Brillouin zone boundary.

A typical data set is illustrated in Figure $5 \mathrm{~b}\left(\theta_{\text {int }}=46.5^{\circ}, t=10 \mathrm{~mm}\right)$ and is in excellent agreement with the finite element method (FEM) model predictions [Ansoft HFSS] using the parameters given above. Note that the frequency of the surface mode has exceeded the infinite-length cut off frequency of the waveguides (solid vertical line, Eq. 1), and appears to have approached that associated with the finite length tubes (broken line, Eq. 2). However the net power flow across the surface (Fig. 5c) suggests that the mode has not yet reached its true asymptotic value (at which $d v / d k=0$ ), a result that becomes evident below when we determine the full dispersion curve (Fig. 5d). By recording a series of reflectivity spectra for different tunnel gaps $t$ for a fixed angle of incidence, we determine the resonant frequency at the gap spacing for which coupling to the mode is strongest, and then repeat the procedure for a number of different angles (circles). In the same manner we extract the optimum coupling frequencies from the reflectivity spectra predictions of the FEM model (solid gray line). At the largest angles, both experiment and modelled data exhibit a small decrease in the resonant frequency with increasing wavevector. These points correspond to the smallest tunnel gaps (typically only a few millimetres for $\theta \sim 55^{\circ}$ ) for which the penetration of the resonant fields into the high index prism becomes significant. Ideally, rather than recording the resonant frequency at the optimum coupling condition, one would increase the tunnelling gap $t$ and note the limiting frequency at which the influence of the prism is no longer significant. However coupling to the mode is often so weak in this limit that it makes accurate determination of the frequency impossible. Therefore we need to confirm the experimental validity of determining the dispersion via the optimum coupling condition. This is satisfactorily achieved by modelling the dispersion of the surface mode in the absence of the prism using the Eigen mode solver of the FEM software (Figure 5d: broken line), and comparing this to our experimental result. There is clearly very good agreement. In a subsequent paper, we shall employ a modal matching method to derive a formalism for accurately determining the dispersion of the surface modes analytically, valid for arrays of holes of any size and depth [22].

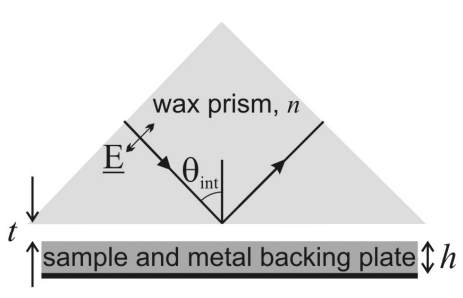

(a)

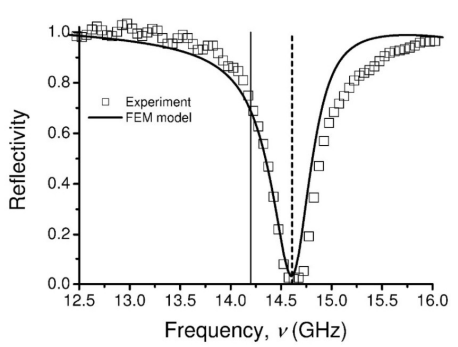

(b)

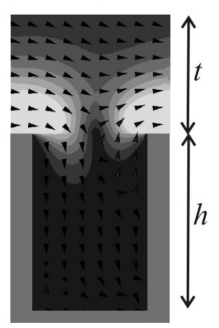

(c)

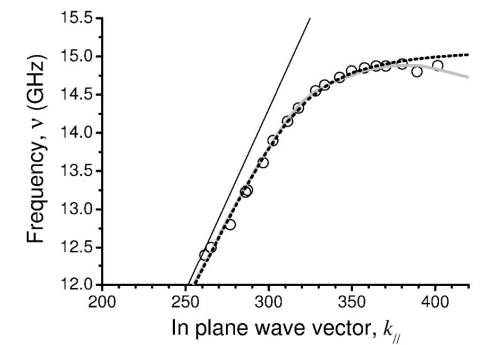

(d)

Fig. 5. (a) Schematic of the experimental set-up. (b) Reflection spectrum from the sample surface for $\theta_{\text {int }}=46.5^{\circ}$, and $45^{\circ}$ between the plane of incidence and the $x z$-plane (squares). The predictions of the FEM model are also shown (line). The solid vertical line corresponds to the cut off frequency of an infinite length guide (Eq. 1) filled with wax and the dimensions previously described. The broken vertical line corresponds to the modified $v_{\mathrm{SP}}$ associated with complete confinement of the waveguide mode to the truncated cavity (Eq. 2). (c) Poynting vector (magnitude and direction) prediction on the plane of incidence of the mode at $14.6 \mathrm{GHz}$. The lightest shading corresponds to power enhancements of at least ten times. (d) Dispersion curves of the surface mode determined from experiment (circles) and numerical models (lines). The predicted data from the optimum coupling condition are shown as a solid grey line, whereas the broken line represents the Eigen mode solution in the absence of the prism.

\subsection{Transmission below cutoff}

Until relatively recently it was believed impossible to transmit a significant intensity of EM radiation through gaps smaller than the illumination wavelength [23]. However, the understanding of the proposed SP-mediated mechanism [24] was put in doubt when numerical predictions [25] confirmed that the phenomenon should still be observed when perfect conductors were used, even though no bound surface wave was thought to exist in this limit. However the aforementioned proposal of Pendry et al. $[19,20]$ was that a structured perfectly conducting surface may support SP-like 
waves that behave in a similar manner to those known to exist on real metals. Here we use microwave transmission measurements and FEM modeling not only to provide further verification of this new class of mode but also to confirm its role in enhanced transmission observed for perforated, perfectly conducting layers [26].

The sample used is once again similar to that used previously, comprised of an array of square cross sectional brass tubes except that each end of the tubes (of height $h=15 \mathrm{~mm}$ ) is left open (Fig. 6a). With EM radiation normally incident a transmission peak $(\sim 23 \mathrm{GHz})$ that is associated with the lowest frequency standing wave resonance of the $\mathrm{TE}_{10}$ waveguide mode of the tube is observed (Fig. 6b,c,d, labeled as mode (1). This frequency therefore presents a limit below which only evanescent fields can exist within the tubes. However an increase of the angle of incidence $(\theta)$ in the $x z$ plane results in a reduction of the frequency of the transmission peak. The EM fields (Fig. 6d) of the resonance labeled as (2) $\left(\theta=26^{\circ}, 21.4 \mathrm{GHz}\right)$ are markedly different in character from those of (1) $\left(\theta=5^{\circ}, 22.8 \mathrm{GHz}\right)$. The former show strongly enhanced fields at the illuminated surface of the array that exponentially decay along the length of the cavity before strengthening again close to the bottom face of the structure. This field distribution is associated with the excitation of a diffractively coupled SP-like mode on the upper surface whose fields decay along the length of the waveguide and couple to an identical mode on the lower face that, in turn, diffractively couples out to free radiation.

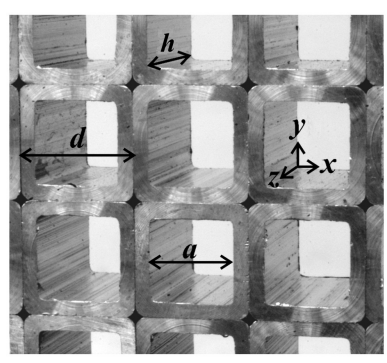

(a)

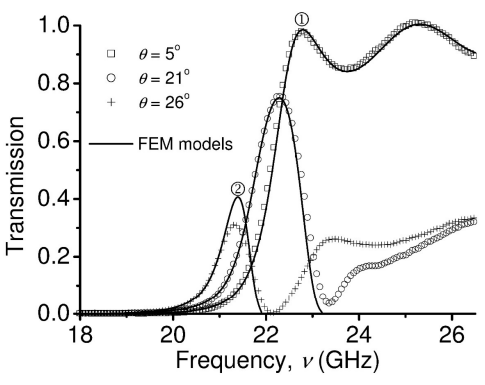

(c)

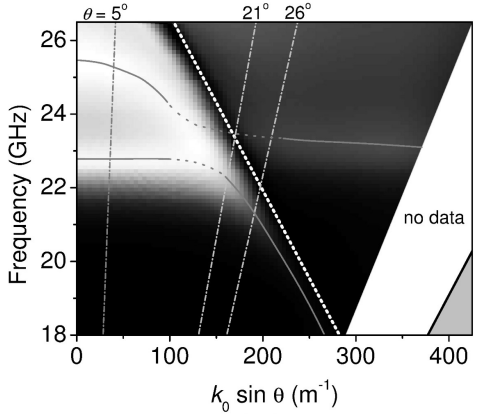

(b)
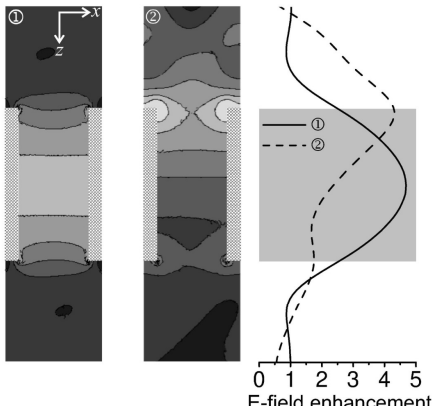

(d)

Fig. 6. (a) Photograph of part of the experimental sample. (b) Intensity of the non-diffracted transmitted beam (greyscale plotted on a linear scale: black $T=0.0$, white $T=1.0$ ) as a function of frequency and in-plane wavevector. The shaded region in the bottom right-hand corner corresponds to momentum-space within which modes may not be directly coupled to as they are beyond the light line (solid black line). The white dotted line represents the first-order diffracted light line associated with the periodicity of the array. Data is only recorded for angles of incidence up to $50^{\circ}$. As a guide to the eye, the solid grey lines provide a trace of the position of the transmission peaks associated with each mode. The dotted segments provide an estimation of the band structure in the region where the transmission peaks are not a good indication of the mode position. (c) Non-diffracted transmission spectra recorded from the sample at angles of $\theta=5,21$ and $26^{\circ}$ (illustrated by grey dot-dash lines in (b)), together with the predictions from the numerical model. In order to reduce the complexity of the problem, model solutions are only requested at frequencies below the onset of diffraction (at $29.0 \mathrm{GHz}, 23.2 \mathrm{GHz}$ and $21.9 \mathrm{GHz}$ for $\theta=5^{\circ}, 21^{\circ}$ and $26^{\circ}$ respectively). (d) Predictions of the timeaveraged electric field enhancements of the resonances labeled (1) $\left(\theta=5^{\circ}, 22.8 \mathrm{GHz}\right)$ and (2) $\left(\theta=26^{\circ}, 21.4 \mathrm{GHz}\right)$. In these grayscale plots, the lightest shading corresponds to field enhancements of six-times and greater. TM-polarized radiation is incident in the $x z$-plane from above. The line graph illustrates the same quantities plotted along a line in the $z$-direction through the centre of a tube. The shaded area corresponds to the region inside the cavity. 
This study has enabled us to resolve a long-standing debate in the literature, proving beyond doubt that plasmon-like surface modes are responsible for the "anomalous" transmission phenomenon though arrays of holes in metal layers even in the limit of perfect conductivity.

\subsection{Coupled SP-like modes}

Subwavelength slits in metal substrates selectively transmit radiation due to the coupling together of surface modes excited on opposite walls of the slit to yield a family of quantized Fabry-Perot (FP)-like resonances [27]. At microwave frequencies, owing to the near-infinite conductivity of the metal [15], these modes can often be considered as simple surface currents and their mutual coupling results in a transverse electromagnetic (TEM) mode propagating along the slit. As previously discussed, these modes can be made more SP-like in character by perforating the surface on a subwavelength scale and we can create an effective surface plasma frequency in the GHz regime by choosing suitable parameters for the hole radius and permittivity of the filler material. Here coupled plasmon-like modes between two such metamaterial surfaces are explored.

The sample (Fig. 7) is formed by drilling an array of holes (radius $r$, depth $h$ ) into equivalent faces of two aluminumalloy plates. The structured surfaces are then carefully aligned and spaced at their long ends with small pieces of noncompressible polyester film to yield a slit of uniform width. The slit is orientated so that its textured faces are in the $x y$ plane. Microwave radiation is incident in the $x y$-plane at an angle $\theta$ from the normal to the entrance face of the slit, and is polarized with the electric field vector parallel to the $z$ axis. In order to fully appreciate and understand our results, it is vital that we can distinguish between the effects of the photonic structure of the hole array and the more interesting plasmonic-like behavior induced below the cutoff frequency of the holes. The periodic patterning of the walls on which the surface modes propagate creates bands of allowed energies separated by band gaps [28]. The lowest-energy band gap occurs at the Brillouin zone boundary, at which the energy of the lower band edge is suppressed below the crossing of the incident and diffracted light lines $\left(v=c / 2 \lambda_{\mathrm{g}}\right)$. We optimize our structure's design, using a FEM computer model [Ansoft HFSS], setting the effective surface plasma frequency below this lowest-energy band edge. Since the effective surface plasma frequency is dictated by the cutoff frequency of the holes, which for holes of infinite depth is given by

$$
v_{\mathrm{SP}}^{(\mathrm{y})}=\frac{1.841 c}{2 \pi r \sqrt{\varepsilon}},
$$

it is clear that this requirement can be met only by filling the holes with a high-index dielectric $\varepsilon>1$. The chosen final design has seven identical holes of radius $r=1.5 \mathrm{~mm}$ along each metal plate of thickness $t=30 \mathrm{~mm}$. The metal plates are separated from one another by $1 \mathrm{~mm}$. The filling material is a mixture by weight of seven parts Dow Corning Syl$\operatorname{gard}{ }^{\circledR} 170$ fast cure silicone elastomer with three parts titanium dioxide. The real part of its permittivity was determined by filling the $1 \mathrm{~mm}$ gaps between an array of 3-mm-wide and $t=20$-mm-thick metal slats [29] and comparing the computer predictions to the measured transmission data, which showed best agreement when $\mathcal{E}=4.7$.

It is useful to first study the transmission of the sample without filling the holes. In this way, we are able to understand the origins of the photonic band structure without significant influence by any "plasmonic" perturbation since the effective surface plasma frequency is now beyond our frequency range of interest $\left(v_{\mathrm{sp}}=56 \mathrm{GHz}\right)$. Figure $8 \mathrm{a}$ shows the experimentally measured dispersion of the FP resonances determined by recording transmission spectra as a function of the angle of incidence, $\theta$. The right inset shows a line plot $(\theta=0)$ together with the predictions from the FEM model, where the hole radius has been used as fitting parameter. The best fit to this and subsequent data is obtained with $r=1.57 \mathrm{~mm}$. While FP resonances are normally equally spaced in frequency, our data illustrates that this is certainly not true for modes supported between textured surfaces. Indeed, by plotting the resonant frequencies at $\theta=0$ as a function of mode number $N$ (left hand inset, Fig. 8a), one can determine an approximate dispersion of the surface modes with in plane wave vector $k_{x}$. As one would expect, the separation in frequency between FP harmonics, and therefore the group velocity of the coupled mode, reduces as the Brillouin zone boundary (between modes $N=7$ and 8 ) is approached. The band gap induced by the photonic surface is clearly visible. 


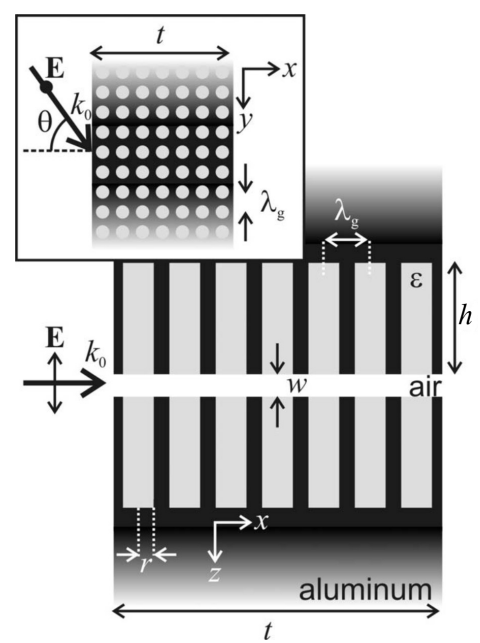

Fig. 7. Schematic illustration of the sample and experimental geometry. The main diagram is drawn on an $x z$-plane that passes through the central axis of the cylindrical holes; the inset is a top view of one of the two substrates.

Now consider the sample with dielectric-filled holes. The comparison between the measured and modeled data is again very good (right hand inset, Figure $8 \mathrm{~b}$ ) where the best fit is obtained with the imaginary part of the permittivity of the filler equal to $0.05 i$. The effective plasma frequency is now $v_{\mathrm{sp}}=26 \mathrm{GHz}$, and above this frequency one observes very little transmission because of absorption associated with waveguide resonances in the filled holes. Now, if we assume that the modes propagating on the textured surfaces inside the slit are SP-like, we would expect the dispersion of the coupled mode to approach $v_{\mathrm{sp}}$ with increasing values of $k_{x}$. Again we plot the resonant frequency of the FP harmonics associated with these coupled modes (left hand inset, Figure 8b) and the dispersion does indeed approach a limiting frequency. However, we are not able to verify the plasmonic nature of the modes based on this evidence alone, owing to the perturbation introduced by the proximity of the modes to the Brillouin zone boundary, but we can plot the dispersion of the modes with $k_{y}=k_{0} \sin \theta$ to provide conclusive evidence.
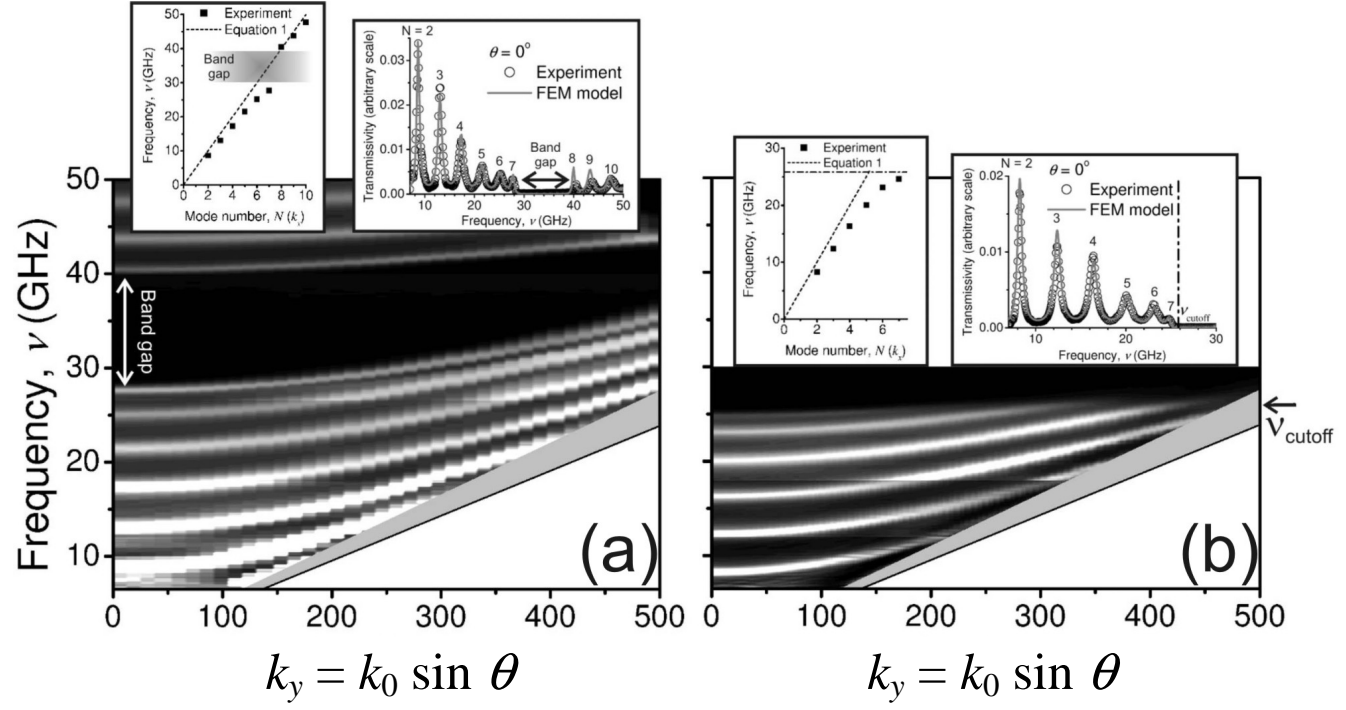

Fig. 8. Experimental transmission data recorded as a function of frequency and $k_{y}=k_{0} \sin \theta$ from the (a) unfilled and (b) filled holes samples ( $\varepsilon=1$ and 4.7 respectively). The black solid line and the gray shaded region correspond to photons at $\theta=90^{\circ}$ and angles beyond the capabilities of the experimental apparatus $\theta>60^{\circ}$, respectively, and the band gap at $\theta=0$ is also marked. Left insets: Dispersion of $\theta=0$ modes with mode number (representative of $k_{x}$ ) Right inset: Comparison of experimental (circles) and modeled (line) data with radiation incident at $\theta=0$. 
Compare the gray scale plots of Figs. 8a and b. For the unfilled sample $\left(\varepsilon=1, v \ll v_{\mathrm{SP}}\right)$ the modes and the lower band edge both show an increase in frequency approximately proportional to $1 / \cos \theta$, which is the same behavior that we expect to observe in the absence of surface patterning. (When $\theta \neq 0, k_{0}$ and $k_{x}$ are not collinear and there is a finite $k_{y}$ component to the incident wave vector $k_{0}=\sqrt{k_{x}^{2}+k_{y}^{2}+k_{z}^{2}}$ where $k_{y}=k_{0} \sin \theta, k_{z}=0$, and from the ideal Fabry-Perot condition [27], $k_{x}=\pi N / t$.) However, for the filled-hole sample $(\varepsilon=4.7)$ one observes a very different response in the character of the higher-order modes. We observe an inflection point in the dispersion curves and an asymptotic approach to the effective surface plasma frequency $v_{\mathrm{sp}} \simeq 26 \mathrm{GHz}$ with increasing $\theta$. This plasmonic-like behavior has been well separated in frequency from the band bending associated with the photonic effects (the lower band edge observed from the unfilled sample is at $\sim 32 \mathrm{GHz}$ for $\theta=30^{\circ}$ ). Furthermore, notice the much longer electric field decay length into the substrate on resonance of the seventh Fabry-Perot harmonic of the elastomer-filled hole sample compared to that of the unfilled sample at a similar frequency (Fig. 9). The magnitude of these fields decreases exponentially into the holes with decay lengths of approximately 2.8 and $1.2 \mathrm{~mm}$, respectively, and is indicative of a reduction of the "effective conductivity" of these metamaterial surfaces.

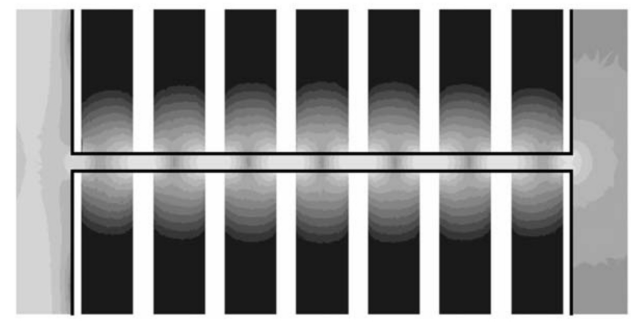

(a)
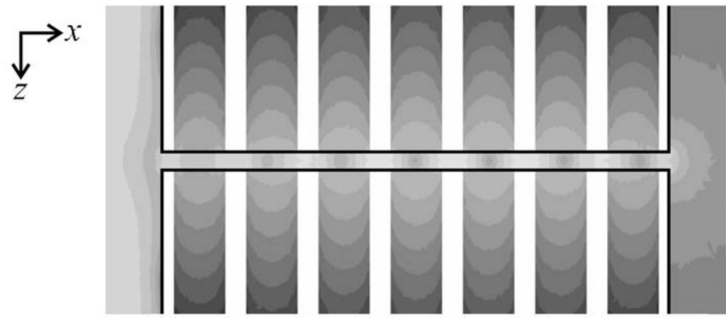

(b)

Fig. 9. FEM model predictions of time-averaged electric field magnitudes on resonance of the seventh Fabry-Perot harmonic of the (a) air-filled, and (b) elastomer-filled hole sample. The plots represent field enhancements, using a logarithmic gray scale between 0.01 and 4, normalized to the strength of the incident field. The microwave beam is incident in the positive $x$ direction, and the fields are plotted on an $x z$-plane of area approximately $37 \times 18 \mathrm{~mm}^{2}$ passing through the central axes of the cylindrical holes. The metal regions are shaded white, and the outline of the structure is highlighted with a black line. The magnitude of the fields decrease exponentially into the holes with a decay length of $\sim 1.2 \mathrm{~mm}$ and $\sim 2.8 \mathrm{~mm}$ for the unfilled and filled hole sample respectively.

The observation of coupled surface-plasmon-like modes between two closely spaced, near-perfectly-conducting metamaterials has been reported [30]. The increase in penetration of the fields close to the cutoff is indicative of a significant reduction of the "effective conductivity" of the structured surfaces.

\section{CONCLUSIONS}

The idea of 'designer' surface modes proposed by Pendry et al. [19] promises the ability to engineer a plasmonic-like surface wave at almost any frequency. We have used the classical methods of grating- and prism-coupling in the microwave regime to study the modes supported by these metamaterials constituting an array of subwavelength holes. We also observe strong resonant transmission of EM radiation below the cutoff frequency of the tubes, and predictions of the EM field on resonance have shown the mode responsible for the subcutoff transmission to be the same plasmonic-like surface wave. Hence our study resolves a long-standing debate, proving beyond doubt that surface modes are responsible for the "anomalous" transmission phenomenon though arrays of holes in metal layers even in the limit of perfect conductivity. Finally we have reported the observation of coupled surface waves between two closely spaced, metamaterial substrates and have observed an increase in penetration of the fields in the plasmonic region, indicative of a significant reduction of the "effective conductivity" of the substrate.

\section{ACKNOWLEDGEMENTS}

The authors would like to acknowledge the financial support of the EPSRC. They would also like to thank Bill Barnes, Euan Hendry, Benjamin Evans and Ian Hooper for useful discussions and their help with these projects, and Peter Cann, Steven Tuckett and Kevin White for machining the experimental samples. 


\section{REFERENCES}

[1] Barlow, H. M. and Cullen, A. L., "Surface waves,” Proc. IEE 100, 329341 (1953).

[2] Goubau, G., "Waves on Interfaces," IRE Trans. Ant. Propag. 7, S140-S146 (1959).

[3] Zenneck, J., "Über die Fortpflanzung ebener elektromagnetischer Wellen längs einer ebenen Leiterflache und ihre Beziehung zur drahtlosen Telegraphie," Ann. Phys. 23, 846-866 (1907).

[4] Wood, R. W., "On a remarkable case of uneven distribution of light in a diffraction grating spectrum," Philos. Mag. 4, 396-402 (1902).

[5] Raether, H., [Surface Plasmons on Smooth and Rough Surfaces and on Gratings], Springer, Berlin (1988).

[6] Fano, U. "Some theoretical considerations on anomalous diffraction gratings," Phys. Rev. 50, 573 (1936).

[7] Ingersoll, L. R., "Polarization of radiation by gratings," Astrophys. J. 51, 129-139 (1920).

[8] Kretschmann, E., and Raether, H., "Radiative decay of nonradiative surface plasmons excited by light," Z. Naturforsch. A 23, 2135-2136 (1968).

[9] Otto, A., "Excitation of nonradiative surface plasma waves in silver by the method of frustrated total reflection," Z. Phys. 216, 398-410 (1968).

[10] Watts, R. A., Sambles, J. R. and Harris, J. B., “An experimental test of conical diffraction theory,” Opt. Commun. 135, 189-192 (1997).

[11] Zhizhin, G. N., Moskalova, M. A., Shomina, E. V. and Yakovlev, V. A.,, [Surface Polaritons: Electromagnetic Waves at Surfaces and Interfaces], North-Holland, Amsterdam (1982).

[12] Hutley, M. C., [Diffraction Gratings], Academic, London, (1982).

[13] Meecham, W. C. and Peters C. W., "Reflection of Plane-Polarized Electromagnetic Radiation from an Echelette Diffraction Grating”, J. Appl. Phys. 28, 216 (1957).

[14] Rayleigh, O. M., “On the dynamical theory of gratings,” Proc. Royal Soc. London. Series A 79, 299-416 (1907).

[15] Suckling, J. R. et al.., "Finite conductance governs the resonance transmission of thin metal slits at microwave frequencies," Phys. Rev. Lett. 92, 147401 (2004).

[16] Palmer, C. H., Evering, F. C. and Nelson, F. M., "Diffraction anomalies for gratings of rectangular profile," Appl. Opt. 4, 1271-1274 (1965).

[17] Hibbins, A. P., Sambles, J. R. and Lawrence, C. R., "Grating-coupled surface plasmons at microwave frequencies," J. Appl. Phys. 86, 1791-1795 (1999)

[18] Pendry, J. B., Holden, A. J., Stewart, W. J. and Youngs, I., "Extremely Low Frequency Plasmons in Metallic Mesostructures," 76, 4773 (1996).

[19] Pendry, J. B., Martín-Moreno, L., and Garcia-Vidal, F. J., "Mimicking Surface Plasmons with Structured Surfaces," Science 305, 847-848 (2004).

[20] Garcia-Vidal, F. J., Martin-Moreno, L. and Pendry, J. B., "Surfaces with holes in them: new plasmonic metamaterials," J. Opt. A 7, S97-S101 (2005).

[21] Hibbins, A. P., Evans, B. R., and Sambles J. R., "Experimental verification of designer surface plasmons", Science 308, 670-672 (2005).

[22] Hibbins, A. P., Hendry, E., Lockyear, M. J. and Sambles, J. R., "Prism Coupling To Plasmon-like Surface Waves on metamaterials" in preparation.

[23] Ebbesen, T. W. et al., "Extraordinary optical transmission through sub-wavelength hole arrays," Nature 391, 667669 (1998).

[24] Sambles, J. R., "More than transparent” Nature 391, 641-642 (1998).

[25] Martín-Moreno, L. et al. "Theory of Extraordinary Optical Transmission through Subwavelength Hole Arrays" Phys. Rev. Lett. 86, 1114-1117 (2001)

[26] Hibbins, A. P., Lockyear, M. J., Hooper I. R. and Sambles J. R., "Waveguide arrays as plasmonic metamaterials: Transmission below cutoff', Phys. Rev. Lett. 96, 073904 (2006).

[27] Hibbins, A. P., Lockyear, M. J. and Sambles, J. R., "The resonant electromagnetic fields of an array of metallic slits acting as Fabry-Perot cavities", J. Appl. Phys. 99, 124903 (2006).

[28] Kitson, S. C., Barnes, W. L. and Sambles, J. R., "Full Photonic Band Gap for Surface Modes in the Visible," Phys. Rev. Lett. 77, 2670-2673 (1996).

[29] Yang, F.Z. and Sambles J.R., "Determination of microwave permittivities using a metallic slit," J. Phys. D 35, 3049-3051 (2002).

[30] Hibbins, A. P., Lockyear, M. J. and Sambles JR, "Coupled plasmon-like modes between metamaterial surfaces", Phys. Rev. B 76, 165431 (2007). 